\title{
Stability Results for an Age-Structured SIS Epidemic Model with Vector Population
}

\author{
He-Long Liu, ${ }^{1}$ Jing-Yuan $\mathrm{Yu}^{2}{ }^{2}$ and Guang-Tian $\mathrm{Zhu}^{3}$ \\ ${ }^{1}$ College of Mathematics and Information Science, Xinyang Normal University, Henan 464000, China \\ ${ }^{2}$ Beijing Institute of Information and Control, Beijing 100037, China \\ ${ }^{3}$ Academy of Mathematics and System Science, C.A.S., Beijing 100080, China
}

Correspondence should be addressed to He-Long Liu; liuhelong2013@126.com

Received 15 November 2014; Revised 13 January 2015; Accepted 15 January 2015

Academic Editor: Zhidong Teng

Copyright (C) 2015 He-Long Liu et al. This is an open access article distributed under the Creative Commons Attribution License, which permits unrestricted use, distribution, and reproduction in any medium, provided the original work is properly cited.

\begin{abstract}
We formulate an age-structured SIS epidemic model with periodic parameters, which includes host population and vector population. The host population is described by two partial differential equations, and the vector population is described by a single ordinary differential equation. The existence problem for endemic periodic solutions is reduced to a fixed point problem of a nonlinear integral operator acting on locally integrable periodic functions. We obtain that if the spectral radius of the Fréchet derivative of the fixed point operator at zero is greater than one, there exists a unique endemic periodic solution, and we investigate the global attractiveness of disease-free steady state of the normalized system.
\end{abstract}

\section{Introduction}

Some infectious diseases such as malaria, Chagas disease, and dengue fever are transmitted via vector. The seasonality of vector population decides that the transmission of infectious diseases is periodic. So, in order to model the seasonal spread of infectious diseases many authors have studied differential equations systems with periodic parameters [1-6].

In order to reflect the effect of demographic behavior of individuals, scholars have recognized that age-structured epidemic models are more realistic, since any disease prevention policy depends on the age structure of host population, and instantaneous death and infection rates depend on the age. Since the pioneer work of McKendrick [3], many authors have studied various age-structured epidemic models [2, 7-12]. However, due to their relatively complex form, the analyses of mathematical properties are difficult especially in the local and global stability of steady states.

The model we study in this paper is an age-structured SIS epidemic model with vector population, host population is divided into two compartments, susceptibles and infectives, and we assume that recovered individuals cannot obtain immunity and directly go back to the susceptibles. The vector population is divided into two groups, susceptibles and infectives. Generally speaking, the number of susceptible population is much greater than the number of infective population in a vector population. So, we may assume that the change of infectives' size does not affect the total number of susceptible vector population.

The age-structured SIS epidemic models have been studied in $[8,9,12-14]$. The general form of these models to a periodic system was given in [6]; the paper analyzed age structured SIS models with seasonal periodicities and vertical transmission and studied the global stability of a nontrivial endemic periodic solution.

The age-structured epidemic models with vector population have been studied in [15-18]. In [15], authors proved that the population dynamics of malaria, formulated vectorhost model for malaria, and used the system of ordinary differential equations to describe the model. Paper [16] discussed a vector-host model for the spread of Chagas disease with infection-age. In [17], a deterministic model showed that the age-structured model underwent the phenomenon of backward bifurcation at $R_{0}=1$ under certain conditions and that the backward bifurcation feature was caused by malariainduced mortality in humans. In [18], authors studied 
the existence and uniqueness of endemic periodic solution of an age-structured SIS epidemic model with periodic parameters.

In our model, host population is described by two partial differential equations, and infective vector population is described by a single ordinary differential equation. Integrating the partial differential equations along the characteristic lines, we can normalize them to a partial differential equation of fraction of infected population and get an expression of the infective host population. Integrating ordinary differential equation, we obtain an expression of the infected vector population. Using these expressions, we can obtain an integral equation, which is a fixed point equation in locally integrable time-periodic functions. From the fixed point theory, we obtain that there exists a unique endemic periodic solution under certain conditions and investigate the global attractiveness of disease-free steady state of the normalized system.

This paper is organized as follows: Section 2 introduces an age-structured SIS epidemic model with vector population. In Section 3, we show the well-posedness of the time evolution problem. In Section 4, we prove the existence of endemic periodic solution of the system in case that threshold value is greater than one. In Section 5, we get that the nontrivial solution is unique if threshold value is greater than one. In Section 6, we study the global attractiveness of infection-free state $E_{0}$. Section 7 contains some discussions of the results.

\section{The Model}

In this section, we formulate an age-structured SIS model, which includes host population and vector population. The host population is divided into two classes: susceptible and infective. Let $S(a, t)$ and $I(a, t)$ be the age-densities of, respectively, the susceptible and infective host population at time $t$. In the vector population, we assume the number of susceptible individuals is far greater than the number of infective individuals, and let $M(t)$ (known $T$-periodic function) be the number of susceptible population and $V(t)$ the number of infective population. Let $N(a)=S(a, t)+I(a, t)$ be the density with respect to age of the total number of the host population and satisfy

$$
N(a)=\mu^{*} N e^{-\int_{0}^{a} \mu_{1}(\sigma) d \sigma}
$$

where the constant $N$ is the total size of the host population, $\mu^{*}$ is the crude death rate of the host population, and $\mu_{1}(a)$ denotes the instantaneous death rate at age $a$ of the host population, is nonnegative, locally integrable on $\left[0, r_{m}\right)\left(r_{m}<\right.$ $+\infty$ denotes the maximum attainable age), and satisfies

$$
\int_{0}^{r_{m}} \mu_{1}(\sigma) d \sigma=+\infty
$$

The crude death rate of the host population is determined such that

$$
\mu^{*} \int_{0}^{r_{m}} f(a) d a=1
$$

where $f(a)=e^{-\int_{0}^{a} \mu_{1}(\sigma) d \sigma}$ is the survival function. We have the relation

$$
N(a)=\mu^{*} N f(a)
$$

Let $\delta_{1}$ be the number of bites per vector per unit time and $c$ be the proportion of infected bites that give rise to infection. Then the force of infection for the host population is defined by

$$
\delta_{1} c V(t)=\eta V(t), \quad \eta=\delta_{1} c .
$$

Let $\delta_{2}$ be the proportion of bites to infected hosts that give rise to infection in vector. Then the number of new infection of vectors per unit time from infected hosts is given by

$$
\begin{array}{r}
\delta_{1} \delta_{2} \frac{\int_{0}^{r_{m}} I(a, t) d a}{N} M(t)=\delta \frac{\int_{0}^{r_{m}} I(a, t) d a}{N} M(t), \\
\delta=\delta_{1} \delta_{2} .
\end{array}
$$

Let $\gamma(a, t)$ be the age-specific recovery rate in the host population and $\mu_{2}(t)$ the per capita death rate of vectors. Biologically speaking, $\gamma(a, t)$ and $\mu_{2}(t)$ are periodic in time $t$, maybe different in period. But for theoretical analysis, we assume that their periods are the same. Moreover we assume that the death rate of the host population is not affected by the presence of the disease.

With these assumptions, we obtain the following system of equations which describes the dynamics of the vector-host model:

$$
\begin{aligned}
& \frac{\partial S(a, t)}{\partial t}+\frac{\partial S(a, t)}{\partial a} \\
& =-\left(\mu_{1}(a)+\eta V(t)\right) S(a, t)+\gamma(a, t) I(a, t), \\
& \frac{\partial I(a, t)}{\partial t}+\frac{\partial I(a, t)}{\partial a} \\
& =\eta V(t) S(a, t)-\left(\mu_{1}(a)+\gamma(a, t)\right) I(a, t), \\
& \frac{d V(t)}{d t}=\delta \frac{\int_{0}^{r_{m}} I(a, t) d a}{N} M(t)-\mu_{2}(t) V(t),
\end{aligned}
$$

with boundary and initial conditions:

$$
\begin{gathered}
S(0, t)=\mu^{*} N, \quad I(0, t)=0, \\
S(a, 0)=S_{0}(a), \quad I(a, 0)=I_{0}(a), \quad V(0)=V_{0},
\end{gathered}
$$

where

$$
\begin{gathered}
S_{0}(a) \geq 0, \quad I_{0}(a) \geq 0, \quad V_{0} \geq 0, \\
S_{0}(a)+I_{0}(a)=N(a) .
\end{gathered}
$$

To simplify the model, let

$$
S(a, t)=f(a) \mu^{*} N s(a, t), \quad I(a, t)=f(a) \mu^{*} N i(a, t) .
$$


The system (7) and (8) can be rewritten as

$$
\begin{aligned}
& \frac{\partial s(a, t)}{\partial t}+\frac{\partial s(a, t)}{\partial a}=-\eta V(t) s(a, t)+\gamma(a, t) i(a, t), \\
& \frac{\partial i(a, t)}{\partial t}+\frac{\partial i(a, t)}{\partial a}=\eta V(t) s(a, t)-\gamma(a, t) i(a, t), \\
& \frac{d V(t)}{d t}=\delta \mu^{*} \int_{0}^{r_{m}} f(a) i(a, t) d a M(t)-\mu_{2}(t) V(t),
\end{aligned}
$$

with boundary and initial conditions:

$$
\begin{gathered}
s(0, t)=1, \quad i(0, t)=0, \\
s(a, 0)=s_{0}(a), \quad i(a, 0)=i_{0}(a), \quad V(0)=V_{0} .
\end{gathered}
$$

The following results will be used in Section 3.

From the third equation of the system (11), we have

$$
\frac{d V(t)}{d t} \leq \delta M(t)-\mu_{2}(t) V(t)
$$

Integrating differential inequality (13), we have

$$
V(t) \leq V_{0} e^{-\int_{0}^{t} \mu_{2}(\sigma) d \sigma}+\delta e^{-\int_{0}^{t} \mu_{2}(\sigma) d \sigma} \int_{0}^{t} e^{\int_{0}^{\tau} \mu_{2}(\sigma) d \sigma} M(\tau) d \tau .
$$

Let $t=m T+t^{\prime}, 0 \leq t^{\prime}<T, m \in N^{+}$. Since $M(t), \mu_{2}(t)$ are $T$-periodic functions, we obtain

$$
\begin{aligned}
V(t) \leq & V_{0} e^{-\int_{0}^{m T} \mu_{2}(\sigma) d \sigma} \\
& +\delta e^{-\int_{0}^{m T} \mu_{2}(\sigma) d \sigma} \int_{0}^{(m+1) T} e^{\int_{0}^{\tau} \mu_{2}(\sigma) d \sigma} M(\tau) d \tau \\
\leq & V_{0} e^{-m \int_{0}^{T} \mu_{2}(\sigma) d \sigma} \\
& +\delta \int_{0}^{T} e^{\int_{0}^{\tau} \mu_{2}(\sigma) d \sigma} M(\tau) d \tau \frac{1-e^{-m \int_{0}^{T} \mu_{2}(\sigma) d \sigma}}{e^{\int_{0}^{T} \mu_{2}(\sigma) d \sigma}-1} \\
\leq & V_{0}+\int_{0}^{T} e^{\int_{0}^{\tau} \mu_{2}(\sigma) d \sigma} M(\tau) d \tau \frac{\delta}{e^{\int_{0}^{T} \mu_{2}(\sigma) d \sigma}-1}<+\infty .
\end{aligned}
$$

\section{Existence and Uniqueness of Solution}

From the first equation and the second equation of the system (11) and (12), we obtain that $s(a, t)+i(a, t)=1$. So, the system (11) and (12) can be reduced to two equations for $i(a, t)$ and $V(t)$ as

$$
\begin{gathered}
\frac{\partial i(a, t)}{\partial t}+\frac{\partial i(a, t)}{\partial a}=\eta V(t)(1-i(a, t))-\gamma(a, t) i(a, t), \\
\frac{d V(t)}{d t}=\delta \mu^{*} \int_{0}^{r_{m}} f(a) i(a, t) d a M(t)-\mu_{2}(t) V(t),
\end{gathered}
$$

with boundary and initial conditions:

$$
\begin{gathered}
i(0, t)=0, \\
i(a, 0)=i_{0}(a), \quad V(0)=V_{0} .
\end{gathered}
$$

We consider the initial-boundary value problem of the system (16) and (17) as an abstract Cauchy problem:

$$
\begin{aligned}
& x^{\prime}(t)=A x+F(t, x), \\
& x(0)=\left(i_{0}(a), V_{0}\right)^{T},
\end{aligned}
$$

where

$$
\begin{gathered}
x=\left(x_{1}, x_{2}\right)^{T}=(i(a, t), V(t))^{T}, \\
A: D(A) \longrightarrow X, \\
A x=\left(\begin{array}{c}
-\frac{d x_{1}}{d a} \\
-x_{2}
\end{array}\right), \\
D(A)=\left\{x, x_{1} \in W^{1}[0,+\infty), x_{2} \in R, x_{1}(0)=0\right\}, \\
X=L^{1}\left[0, r_{m}\right) \times R,
\end{gathered}
$$

endowed with the norm

$$
\begin{aligned}
\|x\|=\left\|x_{1}\right\|+\left|x_{2}\right|, \quad\left\|x_{1}\right\| & =\int_{0}^{r_{m}}\left|x_{1}(a)\right| d a, \\
x & =\left(x_{1}, x_{2}\right)^{T} \in X .
\end{aligned}
$$

Suppose $\gamma_{1}(a, t), \mu_{1}(a) \in L^{\infty}[0,+\infty)$; we define

$$
F(t, x)=\left(\begin{array}{c}
F: X \rightarrow X \\
\eta x_{2}\left(1-x_{1}\right)-\gamma(a, t) x_{1} \\
\delta \mu^{*} \int_{0}^{r_{m}} f(a) x_{1}(a) d a M(t)-\left(\mu_{2}(t)-1\right) x_{2}
\end{array}\right) .
$$

The system (18) is a semilinear nonautonomous Cauchy problem, we easily obtain that the operator $A$ is the infinitesimal generator of $C_{0}$-semigroup $T(t), t \geq 0$, and $F$ is continuously Fréchet differentiable on $X$. Then for each $x_{0} \in D(A)$, there exists a maximal interval of existence $[0, m)$ and a unique mild solution $t \rightarrow x\left(t, x_{0}\right)$ (see $[8,14,19]$ ), which satisfies (18), where either $m=+\infty$ or $m<+\infty$, in the case $\lim _{t \rightarrow m}\left\|x\left(t, x_{0}\right)\right\|=\lim _{t \rightarrow m}(\|i(a, t)\|+|V(t)|)=+\infty$. Since $i(a, t) \leq 1$, and from (15) $V(t)<+\infty$, we easily obtain $m=+\infty$. So, we have the following theorem.

Theorem 1. The initial-boundary value problem (18), that is, the system (16) and (17), has a unique nonnegative mild solution $x=(i(a, t), V(t))^{T}$.

\section{Existence of Endemic Periodic Solution}

In this section, we discuss existence of endemic periodic solution of the system (16) and (17). Let $X_{T}=X_{1, T} \times X_{2, T}$, 
where $X_{1, T}$ is the set of locally integrable $T$-periodic $L^{1}\left[0, r_{m}\right)$-valued functions with norm

$$
\begin{array}{r}
\left\|x_{1}\right\|_{X_{1, T}}=\int_{0}^{T}\left\|x_{1}(t)\right\| d t=\int_{0}^{T} \int_{0}^{r_{m}}\left|x_{1}(a, t)\right| d a d t, \\
x_{1} \in X_{1, T},
\end{array}
$$

$X_{2, T}$ is the set of locally integrable $T$-periodic functions with norm

$$
\left\|x_{2}\right\|_{X_{2, T}}=\int_{0}^{T}\left|x_{2}(t)\right| d t, \quad x_{2} \in X_{2, T}
$$

and $X_{T}^{+}=X_{1, T}^{+} \times X_{2, T}^{+} ; X_{T}^{+}, X_{1, T}^{+}, X_{2, T}^{+}$are their positive cone, respectively. We give the state space of the system (16) and (17) as follows:

$$
X_{T}^{*}=\left\{x \in X_{T}^{+}: 0 \leq x_{1}(a, t) \leq 1, x_{2}(t) \geq 0\right\} .
$$

If $\left(i^{*}(a, t), V^{*}(t)\right)^{T} \in X_{T}^{*}-\{0\}$ is an endemic $T$-periodic solution of the system (16) and (17), then it satisfies

$$
\begin{gathered}
\frac{\partial i^{*}(a, t)}{\partial t}+\frac{\partial i^{*}(a, t)}{\partial a} \\
=\eta V^{*}(t)\left(1-i^{*}(a, t)\right)-\gamma(a, t) i^{*}(a, t), \\
\frac{d V^{*}(t)}{d t}=\delta \mu^{*} \int_{0}^{r_{m}} f(a) i^{*}(a, t) d a M(t) \\
-\mu_{2}(t) V^{*}(t), \\
i^{*}(0, t)=0 .
\end{gathered}
$$

Integrating the first equation of (25) along the characteristic lines, we have

$$
i^{*}(a, t)=\eta \int_{0}^{a} V^{*}(t-a+\sigma) e^{-\int_{\sigma}^{a}\left(\eta V^{*}(t-a+\rho)+\gamma(t-a+\rho, \rho)\right) d \rho} d \sigma,
$$

and $0 \leq i^{*}(a, t) \leq 1$.

From the second equation of (25), we obtain

$$
\begin{aligned}
V^{*}(t)= & \mu^{*} \delta e^{-\int_{0}^{t} \mu_{2}(\tau) d \tau} \\
& \cdot\left(\int_{0}^{t} \int_{0}^{r_{m}} f(a) i^{*}(a, \tau) M(\tau) e^{\int_{0}^{\tau} \mu_{2}(\rho) d \rho} d a d \tau\right. \\
& \left.+V_{0}^{*}\right)
\end{aligned}
$$

where $V_{0}^{*}=\int_{0}^{T} \int_{0}^{r_{m}} f(a) i^{*}(a, \tau) M(\tau) e^{\int_{0}^{\tau} \mu_{2}(\rho) d \rho} d a d \tau /$ $\left(e^{\int_{0}^{T} \mu_{2}(\rho) d \rho}-1\right)$.
Substituting (26) into (27), we have

$$
\begin{aligned}
& V^{*}(t)=\mu^{*} \delta \eta e^{-\int_{0}^{t} \mu_{2}(\tau) d \tau} \\
& \cdot\left(\int_{0}^{t} M(\tau) e^{\int_{0}^{\tau} \mu_{2}(\rho) d \rho} \int_{0}^{r_{m}} f(a) \cdot \int_{0}^{a} V^{*}(\tau-a+\sigma)\right. \\
& \cdot e^{-\int_{\sigma}^{a}\left(\eta V^{*}(\tau-a+\rho)+\gamma(\tau-a+\rho, \rho)\right) d \rho} d \sigma d a d \tau \\
& +\frac{1}{e^{\int_{0}^{T} \mu_{2}(\rho) d \rho}-1} \\
& \cdot \int_{0}^{T} M(\tau) e^{\int_{0}^{\tau} \mu_{2}(\rho) d \rho} \int_{0}^{r_{m}} f(a) \cdot \int_{0}^{a} V^{*}(\tau-a+\sigma) \\
& \left.\cdot e^{-\int_{\sigma}^{a}\left(\eta V^{*}(\tau-a+\rho)+\gamma(\tau-a+\rho, \rho)\right) d \rho} d \sigma d a d \tau\right) \\
& =\mu^{*} \delta \eta e^{-\int_{0}^{t} \mu_{2}(\tau) d \tau} \\
& \cdot\left(\int_{0}^{t} M(\tau) e^{\int_{0}^{\tau} \mu_{2}(\rho) d \rho} \int_{0}^{r_{m}} f(a) \cdot \int_{0}^{a} V^{*}(\tau-\sigma)\right. \\
& \cdot e^{-\int_{(a-\sigma)}^{a}\left(\eta V^{*}(\tau-a+\rho)+\gamma(\tau-a+\rho, \rho)\right) d \rho} d \sigma d a d \tau \\
& +\frac{1}{e^{\int_{0}^{T} \mu_{2}(\rho) d \rho}-1} \\
& \cdot \int_{0}^{T} M(\tau) e^{\int_{0}^{\tau} \mu_{2}(\rho) d \rho} \int_{0}^{r_{m}} f(a) \cdot \int_{0}^{a} V^{*}(\tau-\sigma) \\
& \left.\cdot e^{-\int_{(a-\sigma)}^{a}\left(\eta V^{*}(\tau-a+\rho)+\gamma(\tau-a+\rho, \rho)\right) d \rho} d \sigma d a d \tau\right) \\
& =\mu^{*} \delta \eta e^{-\int_{0}^{t} \mu_{2}(\tau) d \tau} \\
& \cdot\left(\eta \int_{0}^{t} M(\tau) e^{\int_{0}^{\tau} \mu_{2}(\rho) d \rho} \int_{0}^{r_{m}} f(a) \cdot \int_{0}^{a} V^{*}(\tau-\sigma)\right. \\
& \cdot e^{-\int_{0}^{\sigma}\left(\eta V^{*}(\tau-\rho)+\gamma(\tau-\rho, a-\rho)\right) d \rho} d \sigma d a d \tau \\
& +\frac{1}{e^{\int_{0}^{T} \mu_{2}(\rho) d \rho-1}} \\
& \cdot \int_{0}^{T} M(\tau) e^{\int_{0}^{\tau} \mu_{2}(\rho) d \rho} \int_{0}^{r_{m}} f(a) \cdot \int_{0}^{a} V^{*}(\tau-\sigma) \\
& \left.\cdot e^{-\int_{0}^{\sigma}\left(\eta V^{*}(\tau-\rho)+\gamma(\tau-\rho, a-\rho)\right) d \rho} d \sigma d a d \tau\right) .
\end{aligned}
$$

According to expression of $V^{*}(t)$, we define a nonlinear positive operator

$$
\begin{aligned}
(\Phi V)(t)= & \mu^{*} \delta \eta e^{-\int_{0}^{t} \mu_{2}(\tau) d \tau} \\
& \cdot\left(\int_{0}^{t} M(\tau) e^{\int_{0}^{\tau} \mu_{2}(\rho) d \rho} \int_{0}^{r_{m}} f(a) \cdot \int_{0}^{a} V(\tau-\sigma)\right.
\end{aligned}
$$




$$
\begin{aligned}
& \cdot e^{-\int_{0}^{\sigma}(\eta V(\tau-\rho)+\gamma(\tau-\rho, a-\rho)) d \rho} d \sigma d a d \tau \\
&+ \frac{1}{e^{\int_{0}^{T} \mu_{2}(\rho) d \rho}-1} \\
& \cdot \int_{0}^{T} M(\tau) e^{\int_{0}^{\tau} \mu_{2}(\rho) d \rho} \int_{0}^{r_{m}} f(a) \cdot \int_{0}^{a} V(\tau-\sigma) \\
&\left.\cdot e^{-\int_{0}^{\sigma}(\eta V(\tau-\rho)+\gamma(\tau-\rho, a-\rho)) d \rho} d \sigma d a d \tau\right)
\end{aligned}
$$

on $X_{2, T}^{+}$.

If $\Phi$ has a nontrivial fixed point $V^{*}=\Phi\left(V^{*}\right), V^{*} \in X_{2, T}^{+}$ $\{0\}$, then from (26) there is a $T$-periodic solution $i^{*}(a, t) \in$ $X_{1, T}^{+}-\{0\}$. So, the system (16) and (17) has an endemic $T$ periodic solution in a weak sense.

In the following, we investigate such a fixed point $V^{*}=$ $\Phi\left(V^{*}\right), V^{*} \in X_{2, T}^{+}-\{0\}$. First, we define a positive bounded linear operator $\Psi: X_{2, T}^{+} \rightarrow X_{2, T}^{+}$as

$$
\begin{aligned}
(\Psi V)(t)=\mu^{*} \delta \eta e^{-\int_{0}^{t} \mu_{2}(\tau) d \tau} & \cdot\left(\int_{0}^{t} M(\tau) e^{\int_{0}^{\tau} \mu_{2}(\rho) d \rho} \int_{0}^{r_{m}} f(a)\right. \\
& \cdot \int_{0}^{a} V(\tau-\sigma) e^{-\int_{0}^{\sigma} \gamma(\tau-\rho, a-\rho) d \rho} d \sigma d a d \tau \\
& +\frac{1}{e^{\int_{0}^{T} \mu_{2}(\rho) d \rho}-1} \\
& \cdot \int_{0}^{T} M(\tau) e^{\int_{0}^{\tau} \mu_{2}(\rho) d \rho} \int_{0}^{r_{m}} f(a) \\
& \left.\cdot \int_{0}^{a} V(\tau-\sigma) e^{-\int_{0}^{\sigma} \gamma(\tau-\rho, a-\rho) d \rho} d \sigma d a d \tau\right)
\end{aligned}
$$

which is the Fréchet derivative of operator $\Phi$ at $V=0$, and it is a majorant of $\Phi$; that is, $\Phi \leq \Psi$.

Next we prove the following lemmas.

Lemma 2. The operator $\Phi$ is monotone nondecreasing and uniformly bounded for $V \in X_{2, T}^{+}$.

Proof. For $V \in X_{2, T}^{+}$, from (29) we obtain

$$
\begin{aligned}
(\Phi V)(t)= & \mu^{*} \delta e^{-\int_{0}^{t} \mu_{2}(\tau) d \tau} \\
& \cdot\left(\int_{0}^{t} M(\tau) e^{\int_{0}^{\tau} \mu_{2}(\rho) d \rho} \int_{0}^{r_{m}} f(a)\right. \\
& \cdot\left(1-e^{-\int_{0}^{a}(\eta V(\tau-\rho)+\gamma(\tau-\rho, a-\rho)) d \rho}\right) d a d \tau
\end{aligned}
$$

$$
\begin{gathered}
-\int_{0}^{t} M(\tau) e^{\int_{0}^{\tau} \mu_{2}(\rho) d \rho} \int_{0}^{r_{m}} f(a) \\
\cdot \int_{0}^{a} \gamma(\tau-\sigma, a-\sigma) \\
\left.\cdot e^{-\int_{0}^{\sigma}(\eta V(\tau-\rho)+\gamma(\tau-\rho, a-\rho)) d \rho} d \sigma d a d \tau\right) \\
+\frac{\mu^{*} \delta e^{-\int_{0}^{t} \mu_{2}(\tau) d \tau}}{e_{0}^{\int_{0}^{T} \mu_{2}(\rho) d \rho}-1} \\
\cdot\left(\int_{0}^{T} M(\tau) e^{\int_{0}^{\tau} \mu_{2}(\rho) d \rho} \int_{0}^{r_{m}} f(a)\right. \\
\cdot\left(1-e^{-\int_{0}^{a}(\eta V(\tau-\rho)+\gamma(\tau-\rho, a-\rho)) d \rho}\right) d a d \tau \\
-\int_{0}^{T} M(\tau) e^{\int_{0}^{\tau} \mu_{2}(\rho) d \rho \int_{0}^{r_{m}}} f(a) \\
\cdot \int_{0}^{a} \gamma(\tau-\sigma, a-\sigma) \\
\left.\cdot e^{-\int_{0}^{\sigma}(\eta V(\tau-\rho)+\gamma(\tau-\rho, a-\rho)) d \rho} d \sigma d a d \tau\right) .
\end{gathered}
$$

From (31), we have that $\Phi$ is monotone nondecreasing and obtain

$$
\begin{aligned}
(\Phi V)(t) \leq & \mu^{*} \delta e^{-\int_{0}^{t} \mu_{2}(\tau) d \tau} \\
& \cdot \int_{0}^{t} M(\tau) e^{\int_{0}^{\tau} \mu_{2}(\rho) d \rho} \int_{0}^{r_{m}} f(a) d a d \tau \\
& +\frac{\mu^{*} \delta e^{-\int_{0}^{t} \mu_{2}(\tau) d \tau}}{e^{\int_{0}^{T} \mu_{2}(\rho) d \rho}-1} \\
& \cdot \int_{0}^{T} M(\tau) e^{\int_{0}^{\tau} \mu_{2}(\rho) d \rho} \int_{0}^{r_{m}} f(a) d a d \tau
\end{aligned}
$$

Since $\mu^{*} \int_{0}^{r_{m}} f(a) d a=1$, similar to (15), we have

$$
(\Phi V)(t) \leq \frac{2 \delta}{e^{\int_{0}^{T} \mu_{2}(\rho) d \rho}-1} \int_{0}^{T} M(\tau) e^{\int_{0}^{\tau} \mu_{2}(\rho) d \rho} d \tau<+\infty
$$

From (33), we obtain that $\Phi$ is uniformly bounded.

Lemma 3. Let $\rho(\Psi)$ be the spectral radius of operator $\Psi$. If $\rho(\Psi)>0$, then it is a positive eigenvalue of $\Psi$ associated with a positive eigenvector $V_{1} \in X_{2, T}^{+}-\{0\}$.

Proof. It is easy to get that $\Psi$ is a linear map from $X_{2, T}^{+}$into itself and leaves the cone invariant. 
Let $(\Psi V)(t)=\left(\Psi_{1} V\right)(t)+\left(\Psi_{2} V\right)(t)$, where

$$
\begin{aligned}
\left(\Psi_{1} V\right)(t)= & \mu^{*} \delta \eta e^{-\int_{0}^{t} \mu_{2}(\tau) d \tau} \\
& \cdot \int_{0}^{t} M(\tau) e^{\int_{0}^{\tau} \mu_{2}(\rho) d \rho} \int_{0}^{r_{m}} f(a) \\
& \cdot \int_{0}^{a} V(\tau-\sigma) e^{-\int_{0}^{\sigma} \gamma(\tau-\rho, a-\rho) d \rho} d \sigma d a d \tau, \\
\left(\Psi_{2} V\right)(t)= & \frac{\mu^{*} \delta \eta e^{-\int_{0}^{t} \mu_{2}(\tau) d \tau}}{e^{\int_{0}^{T} \mu_{2}(\rho) d \rho}-1} \\
& \cdot \int_{0}^{T} M(\tau) e^{\int_{0}^{\tau} \mu_{2}(\rho) d \rho} \int_{0}^{r_{m}} f(a) \\
& \cdot \int_{0}^{a} V(\tau-\sigma) e^{-\int_{0}^{\sigma} \gamma(\tau-\rho, a-\rho) d \rho} d \sigma d a d \tau .
\end{aligned}
$$

$\left(\Psi_{1} V\right)(t)$ can be rewritten as

$$
\begin{aligned}
\left(\Psi_{1} V\right)(t)= & \mu^{*} \delta \eta e^{-\int_{0}^{t} \mu_{2}(\tau) d \tau} \\
& \cdot \int_{0}^{t} M(\tau) e^{\int_{0}^{\tau} \mu_{2}(\rho) d \rho} \int_{0}^{r_{m}} \int_{\sigma}^{r_{m}} f(a) \\
& \cdot V(\tau-\sigma) e^{-\int_{0}^{\sigma} \gamma(\tau-\rho, a-\rho) d \rho} d a d \sigma d \tau \\
= & \mu^{*} \delta \eta e^{-\int_{0}^{t} \mu_{2}(\tau) d \tau} \\
& \cdot \int_{0}^{t} M(\tau) e^{\int_{0}^{\tau} \mu_{2}(\rho) d \rho} \int_{\tau-r_{m}}^{\tau} V(\sigma) \int_{\tau-\sigma}^{r_{m}} f(a) \\
& \cdot e^{-\int_{0}^{\tau-\sigma} \gamma(\tau-\rho, a-\rho) d \rho} d a d \sigma d \tau .
\end{aligned}
$$

We extend the domain of $l(a)$ and define $l(a)=0$ for $a<0$ or $a \geq r_{m}$; then we have

$$
\begin{gathered}
\mu^{*} \delta \eta e^{-\int_{0}^{t} \mu_{2}(\tau) d \tau} \int_{0}^{t} M(\tau) e^{\int_{0}^{\tau} \mu_{2}(\rho) d \rho} \int_{-\infty}^{\tau-r_{m}} V(\sigma) \int_{\tau-\sigma}^{r_{m}} f(a) \\
\cdot e^{-\int_{0}^{\tau-\sigma} \gamma(\tau-\rho, a-\rho) d \rho} d a d \sigma d \tau=0, \\
\mu^{*} \delta \eta e^{-\int_{0}^{t} \mu_{2}(\tau) d \tau} \int_{0}^{t} M(\tau) e^{\int_{0}^{\tau} \mu_{2}(\rho) d \rho} \int_{\tau}^{+\infty} V(\sigma) \int_{\tau-\sigma}^{r_{m}} f(a) \\
\cdot e^{-\int_{0}^{\tau-\sigma} \gamma(\tau-\rho, a-\rho) d \rho} d a d \sigma d \tau=0 .
\end{gathered}
$$

From (35) and (36), we have

$$
\begin{aligned}
& \int_{\tau-r_{m}}^{\tau} V(\sigma) \int_{\tau-\sigma}^{r_{m}} f(a) e^{-\int_{0}^{\tau-\sigma} \gamma(\tau-\rho, a-\rho) d \rho} d a d \sigma \\
& \quad=\int_{-\infty}^{+\infty} V(\sigma) \int_{\tau-\sigma}^{r_{m}} f(a) e^{-\int_{0}^{\tau-\sigma} \gamma(\tau-\rho, a-\rho) d \rho} d a d \sigma \\
& =\sum_{n=-\infty}^{+\infty} \int_{n T}^{(n+1) T} V(\sigma) \int_{\tau-\sigma}^{r_{m}} f(a) e^{-\int_{0}^{\tau-\sigma} \gamma(\tau-\rho, a-\rho) d \rho} d a d \sigma
\end{aligned}
$$

$$
\begin{aligned}
& =\sum_{n=-\infty}^{+\infty} \int_{0}^{T} V(\sigma+n T) \int_{\tau-n T-\sigma}^{r_{m}} f(a) \\
& \cdot e^{-\int_{0}^{\tau-n T-\sigma} \gamma(\tau-\rho, a-\rho) d \rho} d a d \sigma \\
& =\int_{0}^{T} V(\sigma) \sum_{n=-\infty}^{+\infty} \int_{\tau-n T-\sigma}^{r_{m}} f(a) e^{-\int_{0}^{\tau-n T-\sigma} \gamma(\tau-\rho, a-\rho) d \rho} d a d \sigma .
\end{aligned}
$$

Combining (35) and (37), we obtain

$$
\begin{aligned}
\left(\Psi_{1} V\right)(t)= & \mu^{*} \delta \eta e^{-\int_{0}^{t} \mu_{2}(\tau) d \tau} \\
& \cdot \int_{0}^{t} M(\tau) e^{\int_{0}^{\tau} \mu_{2}(\rho) d \rho} \\
& \cdot \int_{0}^{T} V(\sigma) \cdot \sum_{n=-\infty}^{+\infty} \int_{\tau-n T-\sigma}^{r_{m}} f(a) \\
& \cdot e^{-\int_{0}^{\tau-n T-\sigma} \gamma(\tau-\rho, a-\rho) d \rho} d a d \sigma d \tau \\
= & \int_{0}^{T} K_{1}(t, \sigma) V(\sigma) d \sigma
\end{aligned}
$$

where

$$
\begin{aligned}
K_{1}(t, \sigma)= & \mu^{*} \delta \eta e^{-\int_{0}^{t} \mu_{2}(\tau) d \tau} \\
& \cdot \int_{0}^{t} M(\tau) e^{\int_{0}^{\tau} \mu_{2}(\rho) d \rho} \\
& \cdot \sum_{n=-\infty}^{+\infty} \int_{\tau-n T-\sigma}^{r_{m}} f(a) e^{-\int_{0}^{\tau-n T-\sigma} \gamma(\tau-\rho, a-\rho) d \rho} d a d \tau .
\end{aligned}
$$

$K_{1}(t, \sigma)$ is well defined and is uniformly bounded; the sum in the expression of $K_{1}(t, \sigma)$ is a finite sum due to $f(a)=0$, $a \leq \tau-n T-\sigma<0$ or $a \geq \tau-n T-\sigma>r_{m}$.

Hence, $\Psi_{1}$ can be regarded as an operator on $L^{1}[0, T]$. From the well-known compactness criteria in $L^{1}$ [20], we obtain that $\Psi_{1}$ is compact.

Similar to $\Psi_{1}$, we get the expression of $\Psi_{2}$ as

$$
\left(\Psi_{2} V\right)(t)=\int_{0}^{T} K_{2}(t, \sigma) V(\sigma) d \sigma,
$$

where

$$
\begin{aligned}
K_{2}(t, \sigma)= & \frac{\mu^{*} \delta \eta e^{-\int_{0}^{t} \mu_{2}(\tau) d \tau}}{e^{\int_{0}^{T} \mu_{2}(\rho) d \rho}-1} \\
& \cdot \int_{0}^{T} M(\tau) e^{\int_{0}^{\tau} \mu_{2}(\rho) d \rho} \\
& \cdot \sum_{n=-\infty}^{+\infty} \int_{\tau-n T-\sigma}^{r_{m}} f(a) e^{-\int_{0}^{\tau-n T-\sigma} \gamma(\tau-\rho, a-\rho) d \rho} d a d \tau,
\end{aligned}
$$


$K_{2}(t, \sigma)$ is well defined and is uniformly bounded, and $\Psi_{2}$ is compact in $L^{1}[0, T]$.

Since $(\Psi V)(t)=\left(\Psi_{1} V\right)(t)+\left(\Psi_{2} V\right)(t)$, we obtain that $\Psi$ is positive, linear, and compact. If $\rho(\Psi)>0$, from the KreinRutman theorem [21], there is $V_{1} \in X_{2, T}^{+}-\{0\}$ such that

$$
\left(\Psi V_{1}\right)(t)=\rho(\Psi) V_{1}(t)
$$

holds.

Using the above lemmas, we have the following theorem.

Theorem 4. If $\rho(\Psi)>1$ holds, then operator $\Phi$ has at least one nontrivial fixed point $V^{*}$ :

$$
V^{*}=\Phi\left(V^{*}\right), \quad V^{*} \in X_{2, T}^{*}-\{0\} ;
$$

that is, the system (16) and (17) has at least an endemic Tperiodic solution.

Proof. From Lemma 3, we have

$$
\begin{aligned}
\left(\Psi V_{1}\right)(t) & =\rho(\Psi) V_{1}(t) \\
& =\int_{0}^{T} K(t, \sigma) V_{1}(\sigma) d \sigma, \quad V_{1}(t) \in X_{2, T}^{*}-\{0\},
\end{aligned}
$$

where $K(t, \sigma)=K_{1}(t, \sigma)+K_{2}(t, \sigma)$. Since $K_{1}(t, \sigma), K_{2}(t, \sigma)$ are uniformly bounded, let

$$
\bar{K}=\sup _{0 \leq t \leq T, 0 \leq \sigma \leq r_{m}} K(t, \sigma)<+\infty .
$$

We have

$$
\rho(\Psi) V_{1}(t) \leq \bar{K} \int_{0}^{T} V_{1}(\sigma) d \sigma=\bar{K}\left\|V_{1}(t)\right\|_{X_{2, T}} .
$$

Let

$$
V^{*}(t)=\frac{\rho(\Psi) \ln \rho(\Psi)}{\eta \bar{K} r_{m}} \frac{V_{1}(t)}{\left\|V_{1}(t)\right\|_{X_{2, T}}} \in X_{2, T}^{*}-\{0\},
$$

whose positivity follows from $\rho(\Psi)>1$. From (29), (44), and (46), we have

$$
\begin{aligned}
\left(\Phi V^{*}\right)(t)= & \mu^{*} \delta \eta e^{-\int_{0}^{t} \mu_{2}(\tau) d \tau} \\
& \cdot\left(\int_{0}^{t} M(\tau) e^{\int_{0}^{\tau} \mu_{2}(\rho) d \rho} \int_{0}^{r_{m}} f(a) \cdot \int_{0}^{a} V^{*}(\tau-\sigma)\right. \\
& \cdot e^{-\int_{0}^{\sigma}\left(\eta V^{*}(\tau-\rho)+\gamma(\tau-\rho, a-\rho)\right) d \rho} d \sigma d a d \tau \\
+ & \frac{1}{e^{\int_{0}^{T} \mu_{2}(\rho) d \rho}-1} \\
& \cdot \int_{0}^{T} M(\tau) e^{\int_{0}^{\tau} \mu_{2}(\rho) d \rho} \int_{0}^{r_{m}} f(a) \cdot \int_{0}^{a} V^{*}(\tau-\sigma) \\
& \left.\cdot e^{-\int_{0}^{\sigma}\left(\eta V^{*}(\tau-\rho)+\gamma(\tau-\rho, a-\rho)\right) d \rho} d \sigma d a d \tau\right)
\end{aligned}
$$

$$
\begin{aligned}
& \geq \mu^{*} \delta \eta e^{-\int_{0}^{t} \mu_{2}(\tau) d \tau} \\
& \cdot\left(\int_{0}^{t} M(\tau) e^{\int_{0}^{\tau} \mu_{2}(\rho) d \rho} \int_{0}^{r_{m}} f(a) \cdot \int_{0}^{a} V^{*}(\tau-\sigma)\right. \\
& \cdot e^{-\left(\ln \rho(\Psi) / \bar{K} r_{m}\left\|V_{1}(t)\right\|_{X_{2, T}}\right) \int_{0}^{r_{m}} \rho(\Psi) V_{1}(\tau-\rho) d \rho} \\
& \cdot e^{-\int_{0}^{\sigma} \gamma(\tau-\rho, a-\rho) d \rho} d \sigma d a d \tau \\
& +\frac{1}{e^{\int_{0}^{T} \mu_{2}(\rho) d \rho}-1} \\
& \cdot \int_{0}^{T} M(\tau) e^{\int_{0}^{\tau} \mu_{2}(\rho) d \rho} \int_{0}^{r_{m}} f(a) \cdot \int_{0}^{a} V^{*}(\tau-\sigma) \\
& \cdot e^{-\left(\ln \rho(\Psi) / \bar{K} r_{m}\left\|V_{1}(t)\right\|_{X_{2, T}}\right) \int_{0}^{r_{m}} \rho(\Psi) V_{1}(\tau-\rho) d \rho} \\
& \left.\cdot e^{-\int_{0}^{\sigma} \gamma(\tau-\rho, a-\rho) d \rho} d \sigma d a d \tau\right) \\
& \geq e^{-\ln \rho(\Psi)}\left(\Psi V^{*}\right)(t) \\
& =V^{*}(t) \cdot
\end{aligned}
$$

From Lemma 2, we obtain that operator $\Phi$ is monotone nondecreasing. So, we define a monotone sequence

$$
V_{n+1}=\Phi\left(V_{n}\right), \quad V_{1} \leq V_{2} \leq \cdots \leq V_{n} \leq \cdots
$$

From Lemma 2, we have that sequence $\left\{V_{n}\right\}$ is bounded above. From B. Levi's theorem, there exists $V^{*}(t) \in X_{2, T}^{*}-\{0\}$ such that $\lim _{n \rightarrow+\infty} V_{n}(t)=V^{*}(t)$ and $V^{*}(t)=\left(\Phi V^{*}\right)(t)$.

\section{Uniqueness of Endemic Periodic Solution}

If $\left(i_{1}^{*}(a, t), V_{1}^{*}(t)\right),\left(i_{2}^{*}(a, t), V_{2}^{*}(t)\right)$ are the endemic $T$-periodic solutions of the system (16) and (17), respectively. Let

$$
G_{j}(t)=\delta \mu^{*} \int_{0}^{r_{m}} f(a) i_{j}^{*}(a, t) d a M(t), \quad j=1,2 .
$$

Since $M(t), i(a, t), j=1,2$ are $T$-periodic functions, then $G_{j}(t), j=1,2$, are periodic functions. From (16), we have

$$
V_{j}^{*}(t)=V_{0, j} e^{-\int_{0}^{t} \mu_{2}(\sigma) d \sigma}+e^{-\int_{0}^{t} \mu_{2}(\sigma) d \sigma} \int_{0}^{t} G_{j}(\tau) e^{\int_{0}^{\tau} \mu_{2}(\sigma) d \sigma} d \tau,
$$

where $V_{0, j}=\left(\int_{0}^{T} \int_{0}^{r_{m}} f(a) i^{*}(a, \tau) M(\tau) e^{\int_{0}^{\tau} \mu_{2}(\rho) d \rho} d a d \tau\right) /$ $\left(e^{\int_{0}^{T} \mu_{2}(\rho) d \rho}-1\right)=\left(\int_{0}^{T} G_{j}(\tau) M(\tau) e^{\int_{0}^{\tau} \mu_{2}(\rho) d \rho} d \tau\right) /\left(e^{\int_{0}^{T} \mu_{2}(\rho) d \rho}-1\right)$, $j=1,2$. 

obtain

Considering that $G_{j}(t), \mu_{2}(t)$ are $T$-periodic functions, we

$$
\begin{aligned}
0<a_{1 j}= & e^{-\int_{0}^{T} \mu_{2}(\sigma) d \sigma} \frac{\int_{0}^{T} G_{j}(\tau) M(\tau) e^{\int_{0}^{\tau} \mu_{2}(\rho) d \rho} d \tau}{e^{\int_{0}^{T} \mu_{2}(\rho) d \rho}-1} \leq V_{j}^{*}(t) \\
\leq & \frac{\int_{0}^{T} G_{j}(\tau) M(\tau) e^{\int_{0}^{\tau} \mu_{2}(\rho) d \rho} d \tau}{e^{\int_{0}^{T} \mu_{2}(\rho) d \rho}-1} \\
& +\int_{0}^{T} G_{j}(\tau) e^{\int_{0}^{\tau} \mu_{2}(\sigma) d \sigma} d \tau \\
= & a_{2 j}<+\infty, \quad j=1,2 .
\end{aligned}
$$

For a number $v \in(0,1)$ and the nonlinear positive operator $\Phi$, we have

$$
\begin{aligned}
& \Phi\left(\nu V_{j}^{*}\right)(t)-\nu \Phi\left(V_{j}^{*}\right)(t) \\
& =\mu^{*} \delta \eta \nu e^{-\int_{0}^{t} \mu_{2}(\tau) d \tau} \\
& \cdot\left(\int_{0}^{t} M(\tau) e^{\int_{0}^{\tau} \mu_{2}(\rho) d \rho} \int_{0}^{r_{m}} f(a)\right. \\
& \cdot \int_{0}^{a} V_{j}^{*}(\tau-\sigma) e^{-\int_{0}^{\sigma} \gamma(\tau-\rho, a-\rho) d \rho} \\
& \cdot\left(e^{-\eta \nu \int_{0}^{\sigma} V_{j}^{*}(\tau-\rho) d \rho}-e^{-\eta \int_{0}^{\sigma} V_{J}^{*}(\tau-\rho) d \rho}\right) d \sigma d a d \tau \\
& +\frac{1}{e^{\int_{0}^{T} \mu_{2}(\rho) d \rho}-1} \\
& \cdot \int_{0}^{T} M(\tau) e^{\int_{0}^{\tau} \mu_{2}(\rho) d \rho} \int_{0}^{r_{m}} f(a) \\
& \cdot \int_{0}^{a} V_{j}^{*}(\tau-\sigma) e^{-\int_{0}^{\sigma} \gamma(\tau-\rho, a-\rho) d \rho} \\
& \left.\cdot\left(e^{-\eta \nu \int_{0}^{\sigma} V_{j}^{*}(\tau-\rho) d \rho}-e^{-\eta \int_{0}^{\sigma} V_{j}^{*}(\tau-\rho) d \rho}\right) d \sigma d a d \tau\right) \\
& =\mu^{*} \delta \eta \nu^{-\int_{0}^{t} \mu_{2}(\tau) d \tau} \\
& \cdot\left(\int_{0}^{t} M(\tau) e^{\int_{0}^{\tau} \mu_{2}(\rho) d \rho} \int_{0}^{r_{m}} f(a) \cdot \int_{0}^{a} V_{j}^{*}(\tau-\sigma)\right. \\
& \cdot e^{-\int_{0}^{\sigma}\left(\eta V_{j}^{*}(\tau-\rho)+\gamma(\tau-\rho, a-\rho) d \rho\right)} \\
& \cdot\left(e^{\eta(1-v) \int_{0}^{\sigma} V_{j}^{*}(\tau-\rho) d \rho}-1\right) d \sigma d a d \tau \\
& +\frac{1}{e^{\int_{0}^{T} \mu_{2}(\rho) d \rho}-1}
\end{aligned}
$$

$$
\begin{gathered}
\cdot \int_{0}^{T} M(\tau) e^{\int_{0}^{\tau} \mu_{2}(\rho) d \rho} \int_{0}^{r_{m}} f(a) \cdot \int_{0}^{a} V_{j}^{*}(\tau-\sigma) \\
\cdot e^{-\int_{0}^{\sigma}\left(\eta V_{j}^{*}(\tau-\rho)+\gamma(\tau-\rho, a-\rho) d \rho\right)} \\
\left.\cdot\left(e^{\eta(1-\nu) \int_{0}^{\sigma} V_{j}^{*}(\tau-\rho) d \rho}-1\right) d \sigma d a d \tau\right)
\end{gathered}
$$

From (52), we have

$$
e^{\eta(1-v) \int_{0}^{\sigma} V_{j}^{*}(\tau-\rho) d \rho}-1 \geq e^{\eta(1-v) a_{1 j} \sigma}-1
$$

Combine (53) and (54) to get

$$
\Phi\left(\nu V_{j}^{*}\right)(t)-\nu \Phi\left(V_{j}^{*}\right)(t) \geq c_{j}>0, \quad j=1,2,
$$

where

$$
\begin{gathered}
c_{j}=\mu^{*} \delta \eta \nu^{-\int_{0}^{t} \mu_{2}(\tau) d \tau} \\
\cdot\left(\int_{0}^{t} M(\tau) e^{\int_{0}^{\tau} \mu_{2}(\rho) d \rho} \int_{0}^{r_{m}} f(a) \cdot \int_{0}^{a} V_{j}^{*}(\tau-\sigma)\right. \\
\cdot e^{-\int_{0}^{\sigma}\left(\eta V_{j}^{*}(\tau-\rho)+\gamma(\tau-\rho, a-\rho) d \rho\right)}\left(e^{\eta(1-\nu) a_{1 j} \sigma}-1\right) d \sigma d a d \tau \\
+\frac{1}{e^{\int_{0}^{T} \mu_{2}(\rho) d \rho}-1} \\
\cdot \int_{0}^{T} M(\tau) e^{\int_{0}^{\tau} \mu_{2}(\rho) d \rho} \int_{0}^{r_{m}} f(a) \cdot \int_{0}^{a} V_{j}^{*}(\tau-\sigma) \\
\cdot e^{-\int_{0}^{\sigma}\left(\eta V_{j}^{*}(\tau-\rho)+\gamma(\tau-\rho, a-\rho) d \rho\right)} \\
\left.\cdot\left(e^{\eta(1-\nu) a_{1 j} \sigma}-1\right) d \sigma d a d \tau\right) .
\end{gathered}
$$

Now we use (52) and (55) to investigate the uniqueness of endemic periodic solution.

From (52) we have

$$
V_{1}^{*}(t) \geq a_{11}=a_{11} a_{22}^{-1} a_{22} \geq a_{11} a_{22}^{-1} V_{2}^{*}(t) .
$$

If $a_{11} a_{22}^{-1}<1$, we take $v_{1}=a_{11} a_{22}^{-1}$. From (55) we have

$$
\Phi\left(v_{1} V_{j}^{*}\right)(t) \geq v_{1} \Phi\left(V_{j}^{*}\right)(t)+c_{j}^{(1)}, \quad c_{j}^{(1)}>0, j=1,2 .
$$

Noting $V_{j}^{*}(t)=\left(\Phi V_{j}^{*}\right)(t), j=1,2$, from Lemma 2 and (52) we obtain

$$
\begin{aligned}
V_{1}^{*}(t) & =\left(\Phi V_{1}^{*}\right)(t) \geq\left(\Phi\left(v_{1} V_{2}^{*}\right)\right)(t) \geq v_{1}\left(\Phi V_{2}^{*}\right)(t)+c_{2}^{(1)} \\
& =v_{1} V_{2}^{*}(t)+c_{2}^{(1)} a_{22}^{-1} a_{22} \geq v_{1} V_{2}^{*}(t)+c_{2}^{(1)} a_{22}^{-1} V_{2}^{*}(t) \\
& =\left(v_{1}+c_{2}^{(1)} a_{22}^{-1}\right) V_{2}^{*}(t) .
\end{aligned}
$$


If $v_{1}+c_{2}^{(1)} a_{22}^{-1}<1$, we take $v_{2}=v_{1}+c_{2}^{(1)} a_{22}^{-1}$ and repeat the above process to have

$$
\begin{aligned}
V_{1}^{*}(t) & \geq\left(\nu_{2}+c_{2}^{(1)} a_{22}^{-1}\right) V_{2}^{*}(t) \\
& =\left(v_{1}+c_{2}^{(1)} a_{22}^{-1}+c_{2}^{(1)} a_{22}^{-1}\right) V_{2}^{*}(t) .
\end{aligned}
$$

Since $\lim _{m \rightarrow+\infty}\left(v_{1}+m c_{2}^{(1)} a_{22}^{-1}\right)=+\infty$, then there exists a number $m_{0} \in N^{+}$such that $\nu_{1}+m_{0} c_{2}^{(1)} a_{22}^{-1} \geq 1$, which implies that

$$
V_{1}^{*}(t) \geq\left(v_{1}+m_{0} c_{2}^{(1)} a_{22}^{-1}\right) V_{2}^{*}(t) .
$$

So, if $a_{11} a_{22}^{-1} \geq 1$ from (57) and if $a_{11} a_{22}^{-1}<1$ from (61), we always have

$$
V_{1}^{*}(t) \geq V_{2}^{*}(t)
$$

Exchanging the role of $V_{1}^{*}(t)$ and $V_{2}^{*}(t)$, similar to the above arguments, we can obtain

$$
\begin{gathered}
V_{2}^{*}(t) \geq a_{12}=a_{12} a_{21}^{-1} a_{21} \geq a_{12} a_{21}^{-1} V_{1}^{*}(t), \\
V_{2}^{*}(t) \leq V_{1}^{*}(t) .
\end{gathered}
$$

Equations (62) and (64) imply that $V_{1}^{*}(t)=V_{2}^{*}(t)$. From (26), we have $i_{1}^{*}(a, t)=i_{2}^{*}(a, t)$.

From Theorem 4 and the above arguments, we obtain the theorem.

Theorem 5. If $\rho(\Psi)>1$ holds, the system (16) and (17) has a unique endemic T-periodic solution in a weak sense.

\section{Stability of the Disease-Free Steady State}

The system (16) always has the infection-free steady state:

$$
E_{0}=\left(i^{*}, V^{*}\right)=(0,0)
$$

In this section, we will prove that the disease-free steady state is global attractiveness. From the first equation of (16), we have

$$
i(a, t)=\left\{\begin{array}{cr}
\eta \int_{0}^{a} e^{-\int_{\sigma}^{a}(\eta V(t-a+\tau)+\gamma(\tau, t-a+\tau)) d \tau} V(t-a+\sigma) d \sigma, & t-a>0, \\
e^{-\int_{0}^{t}(\eta V(a-t+\tau)+\gamma(\tau, a-t+\tau)) d \tau} i_{0}(a-t) & \\
+\eta \int_{0}^{t} e^{-\int_{\sigma}^{t}(\eta V(a-t+\tau)+\gamma(\tau, a-t+\tau)) d \tau} V & \\
\cdot(a-t+\sigma) d \sigma, & a-t \geq 0 .
\end{array}\right.
$$

From the second equation of (16), we have

$$
\begin{aligned}
V(t)= & \mu^{*} \delta e^{-\int_{0}^{t} \mu_{2}(\tau) d \tau} \\
& \cdot\left(\int_{0}^{t} \int_{0}^{r_{m}} f(a) i(a, \tau) M(\tau) e^{\int_{0}^{\tau} \mu_{2}(\rho) d \rho} d a d \tau+V_{0}\right) \\
= & \mu^{*} \delta e^{-\int_{0}^{t} \mu_{2}(\tau) d \tau} \\
& \cdot\left(\int _ { 0 } ^ { t } \left(\int_{0}^{\tau} f(a) i(a, \tau) M(\tau) e^{\int_{0}^{\tau} \mu_{2}(\rho) d \rho} d a d \tau\right.\right. \\
& \left.+\int_{\tau}^{r_{m}} f(a) i(a, \tau) M(\tau) e^{\int_{0}^{\tau} \mu_{2}(\rho) d \rho} d a d \tau\right) \\
& \left.+V_{0}\right) .
\end{aligned}
$$

Substituting (66) into (67), we have

$$
V(t)=\mu^{*} \delta e^{-\int_{0}^{t} \mu_{2}(\tau) d \tau}\left(F_{1}(t)+F_{2}(t)+F_{3}(t)+V_{0}\right),
$$

where

$$
\begin{gathered}
F_{1}(t)=\eta \int_{0}^{t} M(\tau) e^{\int_{0}^{\tau} \mu_{2}(\rho) d \rho} \int_{0}^{\tau} f(a) \\
\cdot \int_{0}^{a} e^{-\int_{\sigma}^{a}(\eta V(\tau-a+\xi)+\gamma(\xi, \tau-a+\xi)) d \xi} \\
\cdot V(\tau-a+\sigma) d \sigma d a d \tau, \\
F_{2}(t)=\int_{0}^{t} M(\tau) e^{\int_{0}^{\tau} \mu_{2}(\rho) d \rho} \int_{\tau}^{r_{m}} f(a) \\
\cdot e^{-\int_{0}^{\tau}(\eta V(a-\tau+\xi)+\gamma(\xi, a-\tau+\xi)) d \xi} i_{0}(a-\tau) \\
F_{3}(t)=\eta \int_{0}^{t} M(\tau) e^{\int_{0}^{\tau} \mu_{2}(\rho) d \rho} \int_{\tau}^{r_{m}} f(a) \\
\cdot \int_{0}^{\tau} e^{-\int_{\sigma}^{\tau}(\eta V(a-\tau+\xi)+\gamma(\xi, a-\tau+\xi)) d \xi} \\
\cdot V(a-\tau+\sigma) d \sigma d a d \tau .
\end{gathered}
$$$$
\cdot e^{-\int_{0}^{\tau}(\eta V(a-\tau+\xi)+\gamma(\xi, a-\tau+\xi)) d \xi} i_{0}(a-\tau) d a d \tau,
$$

For $t>r_{m}$, by variable substitution, $F_{1}(t), F_{2}(t), F_{3}(t)$ can be rewritten as

$$
\begin{array}{r}
F_{1}(t)=\eta \int_{0}^{r_{m}} M(\tau) e^{\int_{0}^{\tau} \mu_{2}(\rho) d \rho} \int_{0}^{\tau} f(a) \\
\cdot \int_{0}^{a} e^{-\int_{0}^{\sigma}(\eta V(\tau-\xi)+\gamma(a-\xi, \tau-\xi)) d \xi}
\end{array}
$$


where

$$
\begin{aligned}
& H(t)=\eta \int_{r_{m}}^{t} M(\tau) e^{\int_{0}^{\tau} \mu_{2}(\rho) d \rho} \int_{0}^{\tau} f(a) \\
& \cdot \int_{0}^{a} e^{-\int_{0}^{\sigma}(\eta V(\tau-\xi)+\gamma(a-\xi, \tau-\xi)) d \xi} \\
& \cdot V(\tau-\sigma) d \sigma d a d \tau, \\
& F_{2}(t)=\int_{0}^{r_{m}} M(\tau) e^{\int_{0}^{\tau} \mu_{2}(\rho) d \rho} \int_{\tau}^{r_{m}} f(a) \\
& \cdot e^{-\int_{0}^{\tau}(\eta V(a-\tau+\xi)+\gamma(\xi, a-\tau+\xi)) d \xi} i_{0}(a-\tau) d a d \tau \\
& +\int_{r_{m}}^{t} M(\tau) e^{\int_{0}^{\tau} \mu_{2}(\rho) d \rho} \int_{\tau}^{r_{m}} f(a) \\
& \cdot e^{-\int_{0}^{\tau}(\eta V(a-\tau+\xi)+\gamma(\xi, a-\tau+\xi)) d \xi} i_{0}(a-\tau) d a d \tau, \\
& F_{3}(t)=\eta \int_{0}^{r_{m}} M(\tau) e^{\int_{0}^{\tau} \mu_{2}(\rho) d \rho} \int_{\tau}^{r_{m}} f(a) \\
& \cdot \int_{0}^{\tau} e^{-\int_{0}^{\sigma}(\eta V(a-\xi)+\gamma(\tau-\xi, a-\xi)) d \xi} \cdot V(a-\sigma) d \sigma d a d \tau \\
& +\eta \int_{r_{m}}^{t} M(\tau) e^{\int_{0}^{\tau} \mu_{2}(\rho) d \rho} \int_{\tau}^{r_{m}} f(a) \\
& \cdot \int_{0}^{\tau} e^{-\int_{0}^{\sigma}(\eta V(a-\xi)+\gamma(\tau-\xi, a-\xi)) d \xi} V(a-\sigma) d \sigma d a d \tau .
\end{aligned}
$$

Noting $f(a)=0, a \geq r_{m}$, for $\tau \geq r_{m}$ we have

$$
\begin{aligned}
& \int_{0}^{\tau} f(a) \int_{0}^{a} e^{-\int_{0}^{\sigma}(\eta V(\tau-\xi)+\gamma(a-\xi, \tau-\xi)) d \xi} V(\tau-\sigma) d \sigma d a \\
& =\int_{0}^{r_{m}} f(a) \int_{0}^{a} e^{-\int_{0}^{\sigma}(\eta V(\tau-\xi)+\gamma(a-\xi, \tau-\xi)) d \xi} V(\tau-\sigma) d \sigma d a \\
& \int_{\tau}^{r_{m}} f(a) e^{-\int_{0}^{\tau}(\eta V(a-\tau+\xi)+\gamma(\xi, a-\tau+\xi)) d \xi} i_{0}(a-\tau) d a=0, \\
& \int_{\tau}^{r_{m}} f(a) \int_{0}^{\tau} e^{-\int_{0}^{\sigma}(\eta V(a-\xi)+\gamma(\tau-\xi, a-\xi)) d \xi} V(a-\sigma) d \sigma d a=0 .
\end{aligned}
$$

From (15) we obtain that $V(t)$ is bounded above. Moreover, from (72) we have

$$
\begin{gathered}
\lim _{t \rightarrow+\infty}\left(\mu^{*} \delta e^{-\int_{0}^{t} \mu_{2}(\tau) d \tau}\left(F_{1}(t)-H(t)\right)\right)=0, \\
\lim _{t \rightarrow+\infty}\left(\mu^{*} \delta e^{-\int_{0}^{t} \mu_{2}(\tau) d \tau} F_{2}(t)\right)=0, \\
\lim _{t \rightarrow+\infty}\left(\mu^{*} \delta e^{-\int_{0}^{t} \mu_{2}(\tau) d \tau} F_{3}(t)\right)=0 .
\end{gathered}
$$

Before proving the global attractiveness of the disease-free steady state, we first prove a lemma.
Lemma 6. Consider

$$
\begin{aligned}
& \lim \sup _{t \rightarrow+\infty}\left(\mu^{*} \delta H(t)\right) \leq \frac{\mu^{*} \delta \eta V^{\infty}}{e^{\int_{0}^{T} \mu_{2}(\rho) d \rho}-1} \\
& \cdot \int_{0}^{T} M(\tau) e^{\int_{0}^{\tau} \mu_{2}(\rho) d \rho \int_{0}^{r_{m}} f(a)} \\
& \cdot \int_{0}^{a} e^{-\int_{0}^{\sigma} \gamma(a-\xi, \tau-\xi) d \xi} d \sigma d a d \tau
\end{aligned}
$$

where $V^{\infty}=\lim \sup _{t \rightarrow+\infty} V(t)$.

Proof. For any given $\varepsilon>0$, we choose $n_{0} \in N_{+}$such that $V(t)<V^{\infty}+\varepsilon$, for $t>n_{0} T-r_{m}$. Let $t=n T+t^{\prime}, 0 \leq t^{\prime}<T$, $n>n_{0}$; we have

$$
\begin{aligned}
& \mu^{*} \delta H(t) \leq \mu^{*} \delta \eta e^{-\int_{0}^{n T} \mu_{2}(\tau) d \tau} \\
& \cdot \int_{r_{m}}^{(n+1) T} M(\tau) e^{\int_{0}^{\tau} \mu_{2}(\rho) d \rho} \int_{0}^{r_{m}} f(a) \\
& \cdot \int_{0}^{a} e^{-\int_{0}^{\sigma} \gamma(a-\xi, \tau-\xi) d \xi} V(\tau-\sigma) d \sigma d a d \tau \\
& \leq \mu^{*} \delta \eta e^{-\int_{0}^{n T} \mu_{2}(\tau) d \tau} \\
& \cdot\left(\int_{r_{m}}^{n_{0} T} M(\tau) e^{\int_{0}^{\tau} \mu_{2}(\rho) d \rho} \int_{0}^{a} f(a) e^{-\int_{0}^{\sigma} \gamma(a-\xi, \tau-\xi) d \xi}\right. \\
& \cdot V(\tau-\sigma) d \sigma d a d \tau+\sup _{n_{0} T-r_{m} \leq t<+\infty} V(t) \\
& \cdot \int_{n_{0} T}^{(n+1) T} M(\tau) e^{\int_{0}^{\tau} \mu_{2}(\rho) d \rho} \int_{0}^{r_{m}} f(a) \\
& \left.\cdot \int_{0}^{a} e^{-\int_{0}^{\sigma} \gamma(a-\xi, \tau-\xi) d \xi} d \sigma d a d \tau\right) \\
& \leq \mu^{*} \delta \eta e^{-\int_{0}^{n T} \mu_{2}(\tau) d \tau} \\
& \cdot\left(\int_{r_{m}}^{n_{0} T} M(\tau) e^{\int_{0}^{\tau} \mu_{2}(\rho) d \rho} \int_{0}^{a} f(a) e^{-\int_{0}^{\sigma} \gamma(a-\xi, \tau-\xi) d \xi}\right. \\
& \cdot V(\tau-\sigma) d \sigma d a d \tau+\left(V^{\infty}+\varepsilon\right) \\
& \cdot \int_{0}^{(n+1) T} M(\tau) e^{\int_{0}^{\tau} \mu_{2}(\rho) d \rho} \int_{0}^{r_{m}} f(a) \\
& \left.\cdot \int_{0}^{a} e^{-\int_{0}^{\sigma} \gamma(a-\xi, \tau-\xi) d \xi} d \sigma d a d \tau\right)
\end{aligned}
$$

Since $M(t), \mu_{2}(t), \gamma(a, t)$ are $T$-periodic functions, we obtain

$$
e^{-\int_{0}^{n T} \mu_{2}(\tau) d \tau}=e^{-n \int_{0}^{T} \mu_{2}(\tau) d \tau}
$$




$$
\begin{gathered}
\int_{0}^{(n+1) T} M(\tau) e^{\int_{0}^{\tau} \mu_{2}(\rho) d \rho} \int_{0}^{r_{m}} f(a) \\
\cdot \int_{0}^{a} e^{-\int_{0}^{\sigma} \gamma(a-\xi, \tau-\xi) d \xi} d \sigma d a d \tau \\
=\frac{e^{n \int_{0}^{T} \mu_{2}(\rho) d \rho}-1}{e^{\int_{0}^{T} \mu_{2}(\rho) d \rho}-1} \\
\cdot \int_{0}^{T} M(\tau) e^{\int_{0}^{\tau} \mu_{2}(\rho) d \rho} \int_{0}^{r_{m}} f(a) \\
\cdot \int_{0}^{a} e^{-\int_{0}^{\sigma} \gamma(a-\xi, \tau-\xi) d \xi} d \sigma d a d \tau .
\end{gathered}
$$

From (75), (76), we take the limit supreme on both sides of (75) when $t \rightarrow+\infty(n \rightarrow+\infty)$ and have

$$
\begin{aligned}
\lim \sup _{t \rightarrow+\infty}\left(\mu^{*} \delta H(t)\right) \leq & \frac{\mu^{*} \delta \eta\left(V^{\infty}+\varepsilon\right)}{e^{\int_{0}^{T} \mu_{2}(\rho) d \rho}-1} \\
& \cdot \int_{0}^{T} M(\tau) e^{\int_{0}^{\tau} \mu_{2}(\rho) d \rho \int_{0}^{r_{m}} f(a)} \\
& \cdot \int_{0}^{a} e^{-\int_{0}^{\sigma} \gamma(a-\xi, \tau-\xi) d \xi} d \sigma d a d \tau .
\end{aligned}
$$

Since $\varepsilon$ is arbitrary, the lemma follows.

Let

$$
\begin{aligned}
R_{0}(T)= & \frac{\mu^{*} \delta \eta}{e^{\int_{0}^{T} \mu_{2}(\rho) d \rho}-1} \\
& \cdot \int_{0}^{T} M(\tau) e^{\int_{0}^{\tau} \mu_{2}(\rho) d \rho} \int_{0}^{r_{m}} f(a) \\
& \cdot \int_{0}^{a} e^{-\int_{0}^{\sigma} \gamma(a-\xi, \tau-\xi) d \xi} d \sigma d a d \tau .
\end{aligned}
$$

Using Lemma 6, we have the following theorem.

Theorem 7. If $R_{0}(T)<1$ holds, then the infection-free steady state $E_{0}$ is global attractiveness; that is,

$$
\lim _{t \rightarrow+\infty}(i(a, t), V(t))=(0,0) .
$$

Proof. From (73) and Lemma 6, we take the limit supreme on both sides of (68) when $t \rightarrow+\infty(n \rightarrow+\infty)$ and have

$$
\begin{aligned}
V^{\infty}=\lim \sup _{t \rightarrow+\infty} V(t) \\
=\lim \sup _{t \rightarrow+\infty}\left(\mu^{*} \delta e^{-\int_{0}^{t} \mu_{2}(\tau) d \tau}\right. \\
\\
\left.\quad \cdot\left(F_{1}(t)+F_{2}(t)+F_{3}(t)+V_{0}\right)\right)
\end{aligned}
$$

$$
\begin{aligned}
& =\lim \sup _{t \rightarrow+\infty}\left(\mu^{*} \delta e^{-\int_{0}^{t} \mu_{2}(\tau) d \tau} H(t)\right) \\
& \leq V^{\infty} R_{0}(T)
\end{aligned}
$$

If $R_{0}(T)<1$, then $V^{\infty}=0$, which implies $\lim _{t \rightarrow+\infty} V(t)=0$. When $t>r_{m} \geq a$, from (66) we have

$$
i(a, t)=\eta \int_{0}^{a} e^{-\int_{\sigma}^{a}(\eta V(t-a+\tau)+\gamma(\tau, t-a+\tau)) d \tau} V(t-a+\sigma) d \sigma .
$$

Since $V^{\infty}=0$, similar to the proof of Lemma 6 , for any given $\varepsilon>0$, we choose $n_{0} \in N_{+}$such that $V(t)<\varepsilon$, for $t>n_{0} T-r_{m}$. From (82) to get

$$
i(a, t) \leq \eta r_{m} \varepsilon
$$

Since $\varepsilon$ is arbitrary, inequality (83) implies

$$
\lim _{t \rightarrow+\infty} i(a, t)=0 \text {. }
$$

This completes our proof.

\section{Discussion}

In this paper, in order to reflect the dependence of vectorborne diseases progress on seasonality of vector population and chronological age of the host population, our SIS epidemic model has periodic parameters, and the host population is structured by the chronological age. So, the SIS epidemic model has a relatively complex form. Due to the difficulty in the analysis, to our knowledge, we do not obtain threshold value for the existence and uniqueness of nontrivial endemic periodic solution of the age-structured SIS epidemic model and do not prove the infection-free state is globally stable and only get the condition under which the infectionfree state is global attractiveness. In addition, for our agedependent case, the stability of the endemic periodic solution $\left(i^{*}(a, t), V^{*}(t)\right)$ for $\rho(\Psi)>1$ has been left as an open problem.

\section{Conflict of Interests}

The authors declare that there is no conflict of interests regarding the publication of this paper.

\section{Acknowledgment}

The authors are grateful to two reviewers for their careful reading of the original manuscript and their many valuable comments and suggestions.

\section{References}

[1] H. W. Hethcote and S. A. Levin, "Periodicity in epidemiological models," in Applied Mathematical Ecology, S. A. Levin, T. G. Hallam, and L. J. Gross, Eds., vol. 18, pp. 193-211, Springer, Berlin, Germany, 1989. 
[2] N. Bacaër and S. Guernaoui, "The epidemic threshold of vectorborne diseases with seasonality," Journal of Mathematical Biology, vol. 53, no. 3, pp. 421-436, 2006.

[3] N. Bacaër, "Approximation of the basic reproduction number R0 for vectorborne diseases with a periodic vector population," Bulletin of Mathematical Biolog, vol. 69, no. 3, pp. 1067-1091, 2007.

[4] W. Wang and X.-Q. Zhao, "Threshold dynamics for compartmental epidemic models in periodic environments," Journal of Dynamics and Differential Equations, vol. 20, no. 3, pp. 699-717, 2008.

[5] Y. Nakata and T. Kuniya, "Global dynamics of a class of SEIRS epidemic models in a periodic environment," Journal of Mathematical Analysis and Applications, vol. 363, no. 1, pp. 230-237, 2010.

[6] M. Langlais and S. N. Busenberg, "Global behaviour in age structured S.I.S. models with seasonal periodicities and vertical transmission," Journal of Mathematical Analysis and Applications, vol. 213, no. 2, pp. 511-533, 1997.

[7] N. Bacaër and R. Ouifki, "Growth rate and basic reproduction number for population models with a simple periodic factor," Mathematical Biosciences, vol. 210, no. 2, pp. 647-658, 2007.

[8] S. N. Busenberg, M. Iannelli, and H. R. Thieme, "Global behavior of an age-structured epidemic model," SIAM Journal on Mathematical Analysis, vol. 22, no. 4, pp. 1065-1080, 1991.

[9] S. N. Busenberg, M. Iannelli, and H. R. Thieme, "Dynamics of an age structured epidemic model," in Dynamical Systems, vol. 4, pp. 1-19, World Scientific, Singapore, 1993.

[10] O. Diekmann, J. A. P. Heesterbeek, and J. A. J. Metz, "On the definition and the computation of the basic reproduction ratio $R_{0}$ in models for infectious diseases in heterogeneous populations," Journal of Mathematical Biology, vol. 28, no. 4, pp. 365-382, 1990.

[11] O. Diekmann, J. A. P. Heesterbeek, and M. G. Roberts, “The construction of next-generation matrices for compartmental epidemic models," Journal of the Royal Society Interface, vol. 7, no. 47, pp. 873-885, 2010.

[12] Z. Feng, W. Huang, and C. Castillo-Chavez, "Global behavior of a multi-group SIS epidemic model with age structure," Journal of Differential Equations, vol. 218, no. 2, pp. 292-324, 2005.

[13] M. Iannelli, F. A. Milner, and A. Pugliese, "Analytical and numerical results for the age-structured S-I-S epidemic model with mixed inter-intracohort transmission," SIAM Journal on Mathematical Analysis, vol. 23, no. 3, pp. 662-688, 1992.

[14] M. Iannelli, M.-Y. Kim, and E.-J. Park, "Asymptotic behavior for an SIS epidemic model and its approximation," Nonlinear Analysis: Theory, Methods \& Applications, vol. 35, no. 7, pp. 797814, 1999.

[15] Z. Feng, D. L. Smith, F. E. McKenzie, and S. A. Levin, "Coupling ecology and evolution: malaria and the $S$-gene across time scales," Mathematical Biosciences, vol. 189, no. 1, pp. 1-19, 2004.

[16] H. Inaba and H. Sekine, "A mathematical model for Chagas disease with infection-age-dependent infectivity," Mathematical Biosciences, vol. 190, no. 1, pp. 39-69, 2004.

[17] F. Forouzannia and A. B. Gumel, "The age-structured model undergoes the phenomenon of backward bifurcation at $R_{0}=1$ under certain conditions," Mathematical Biosciences, vol. 247, pp. 80-94, 2014.

[18] T. Kuniya and H. Inaba, "Endemic threshold results for an age-structured SIS epidemic model with periodic parameters," Journal of Mathematical Analysis and Applications, vol. 402, no. 2, pp. 477-492, 2013.
[19] A. Pazy, Semigroups of Linear Operators and Applications to Partial Differential Equations, vol. 44 of Applied Mathematical Sciences, Springer, New York, NY, USA, 1983.

[20] K. Yosida, Functional Analysis, vol. 123 of Grundlehren der Mathematischen Wissenschaften [Fundamental Principles of Mathematical Sciences], Springer, Berlin, Germany, 6th edition, 1980.

[21] M. G. Krein and M. A. Rutman, "Linear operators leaving invariant a cone in a Banach space," American Mathematical Society Translation, vol. 23, no. 1, pp. 199-325, 1948. 


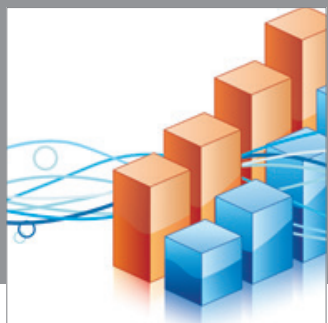

Advances in

Operations Research

mansans

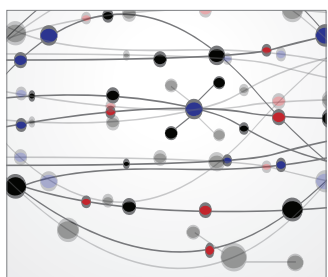

The Scientific World Journal
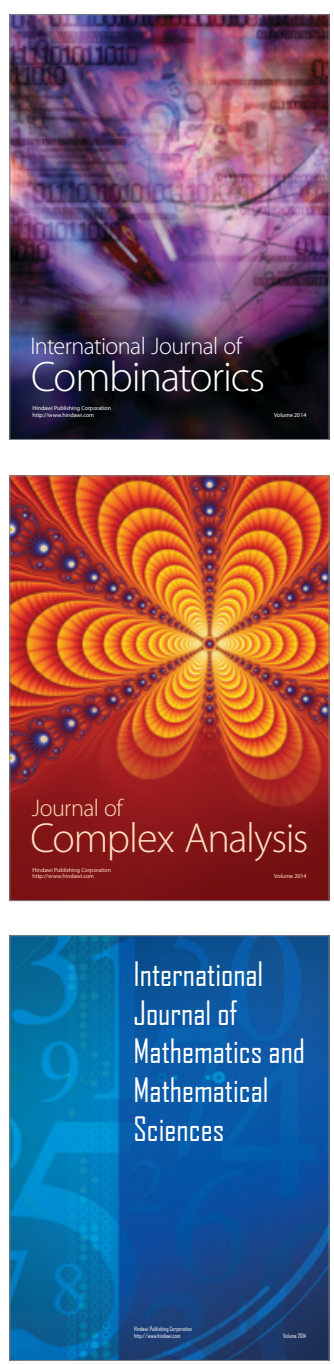
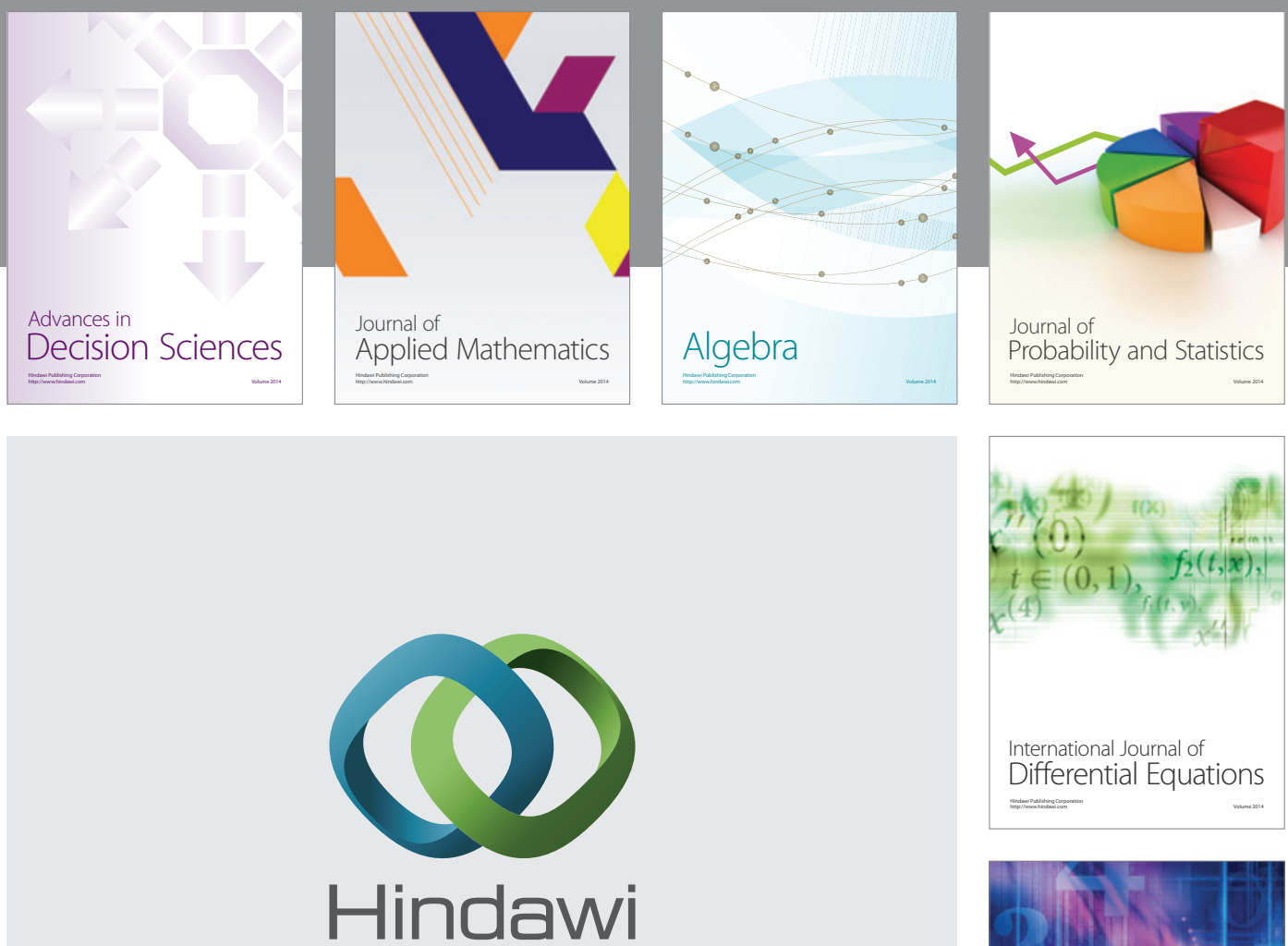

Submit your manuscripts at http://www.hindawi.com
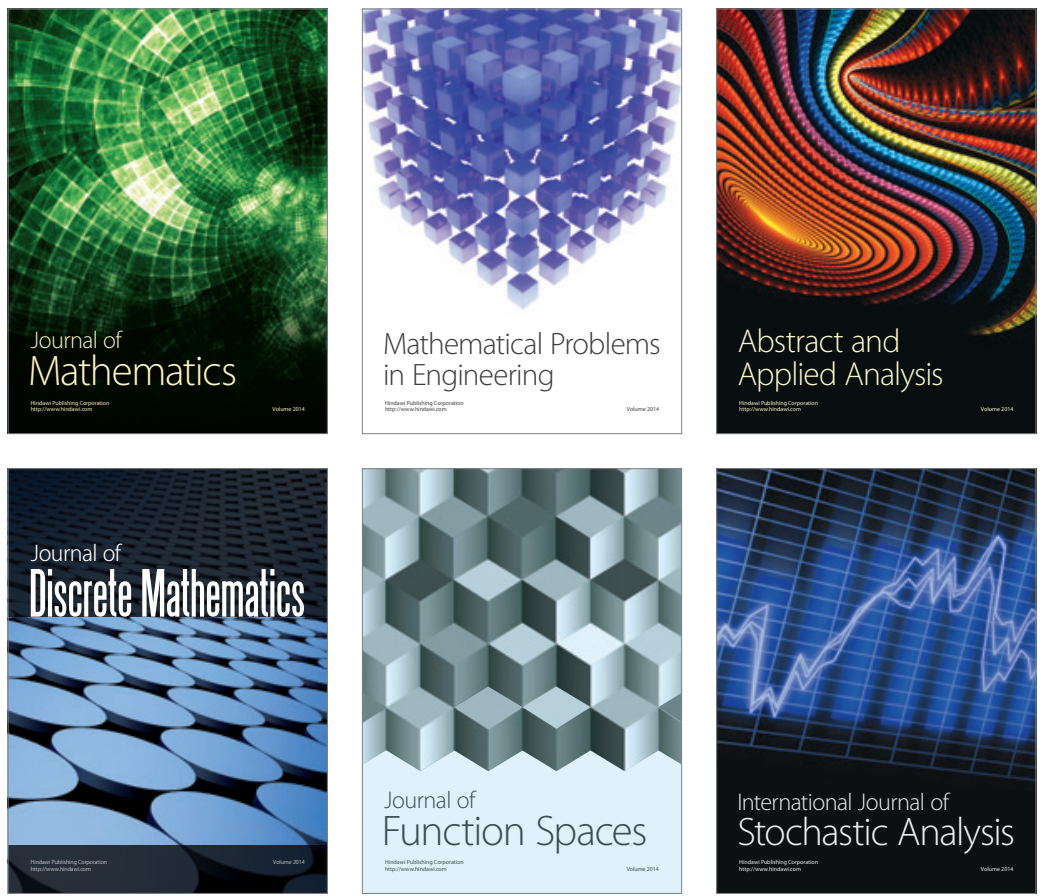

Journal of

Function Spaces

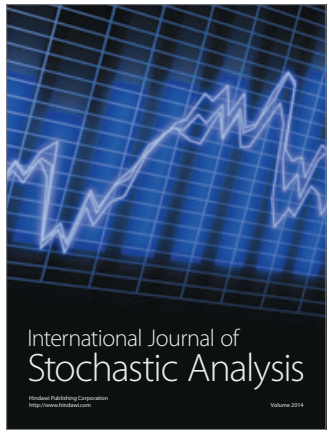

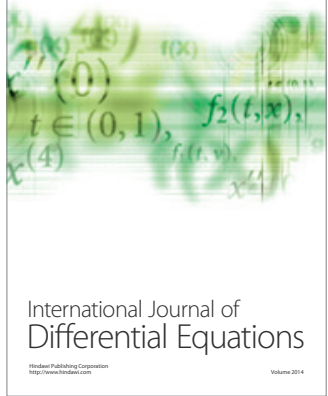
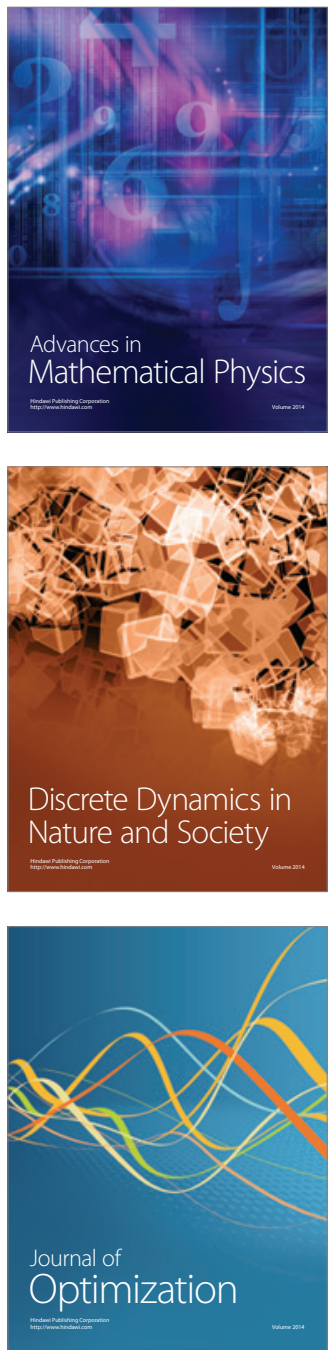